\title{
LOW MOLECULAR WEIGHT HEPARINS
}

\author{
Lt Col SK SHARMA*
}

$\mathbf{H}$ eparin was discovered rather accidentally by $\mathrm{J}$ McLean, a medical student in 1906. It was named heparin as it was thought to be abundant in the liver. It has been used for over fifty years in the acute management of venous thrombosis and pulmonary embolism. Its role has also emerged in arterial thrombotic events particularly in unstable angina, myocardial infarction and acute peripheral arterial occlusion. It was used earlier only in the unfractionated form. One of the major limitations of conventional heparin is that its use requires constant monitoring of activated thromboplastin time (APTT). Consequently it can only be administered to a hospitalized patient. Adverse effects like bleeding, heparin induced thrombocytopenia and osteoporosis on prolonged use may be troublesome. Over the past decade newer heparins, the low molecular weight heparin (LMWH) have emerged. They are prepared from unfractionated heparin by enzymatic or depolymerisation methods (Table 1). They can be given safely on outpatient basis without any monitoring and appear to have fewer side effects [1]. They are already widely replacing heparin in Europe and North America[2].

TABLE 1

Low molecular weight heparin preparations

\begin{tabular}{llc}
\hline Preparation & Method of preparation & Molecular weight \\
\hline 1. Ardeparin & Peroxidative depolymerisation & 6000 \\
2. Dalteparin & Nitrous acid depolymerisation & 6000 \\
3. Enoxaparin & Alkaline depolymerisation & 4200 \\
4. Nadroparin & Nitrous acid depolymerisation & 4500 \\
5. Reviparin & Nitrous acid depolymerisation & 4000 \\
6. Tinzaparin & Heparinase digestion & 4500 \\
\hline
\end{tabular}

\section{Structure and Mechanism of Action}

Like heparin, LMWH are glycosaminoglycans, consisting of chains of alternating residues of D-glucosamines and uronic acid, either glucoronic acid or iduronic acid. Heparin is a heterogenous mixture of polysaccharide chains varying in molecular weight from 3000 to 30,000 . whereas LMWH have chains with a mean molecular weight of 5000 . Both heparin and LMWH exert their anticoagulant activity by activating antithrombin (earlier called antithrombin III), which accelerates the inactivation of coagulation enzymes thrombin (factor IIA), factor Xa and factor IXA. This interaction with antithrombin is mediated by a unique pentasaccharide sequence. Binding of pentasaccharide to antithrombin causes a conformational change in antithrombin that accentuates its action with thrombin and factor $\mathrm{Xa}$ by about a thousand times. To inactivate thrombin, drug must bind to both thrombin and anti thrombin, thereby forming a tertiary complex. This complex can only be formed by pentasaccharides-containing heparin chains comprised of at least 18 saccharide units. Most of the chains of heparin have at least 18 saccharide units, whereas fewer than half of LMWH chains are of this length. More simply put, heparin fragments can only bind to both antithrombin and thrombin when they exceed a molecular weight of 5000 . Fragments of smaller size (LMWH) can not bind to both antithrombin and thrombin, but can bind to antithrombin and factor $\mathrm{Xa}$ and catalyze the inactivation of factor $\mathrm{Xa}$. Consequently heparin has equivalent activity against antithrombin and factor Xa but LMWH has a greater activity against factor $\mathrm{Xa}$.

\section{Pharmacokinetics}

LMWH produce a more predictable anticoagulant response than heparin because of their better bioavailability, longer half life and dose-independent clearance[2]. LMWH have a plasma half life two to four times that of heparin and are mainly eliminated by the kidneys. Elimination is slower and independent of dose. This permits less frequent dosing, Heparin is eliminated in two phases: a rapid saturable phase reflecting hepatic uptake, and a slower phase corresponding to renal clearance. Pharmacokinetic differences between heparin and LMWH are explained by the lesser property of LMWH to bind to plasma proteins, endothelial cells and macrophages compared to heparin. Heparin also binds to platelet factor 4 (released from activated platelets), and high molecular weight multimers of von Willebrand factor. Some of

\footnotetext{
Reader, Medicine, Armed Forces Medical College, Pune 411040.
} 
the heparin binding proteins are acute phase reactants and their concentrations are increased in ill patients, whereas platelet factor 4 and von Willebrand factor are released during the clotting process. This causes an unpredictable anticoagulant response with heparin[3]. All LMWH are recommended to be administered subcutaneously (SC) and monitoring of APTT is not required.

\section{Clinical Uses}

Prophylaxis:Anticoagulants are required to prevent the risk of perioperative deep vein thrombosis and fatal pulmonary embolism after major general surgery, orthopaedic surgery like hip and knee transplants, spinal cord injury and multiple trauma. The risk is increased with increased duration of surgery, increasing age and the presence of co-morbid conditions like coronary artery disease, malignancy and thrombophilic states. LMWH are probably more effective than heparin and warfarin[4]. Dalteparin or Enoxaparin in a dose of 2500 units SC 1 to 2 hours before general surgery followed by once daily for 10 days is recommended in high risk cases. In high risk orthopaedic cases 5000 units SC may be started 12 hours after the surgery.

Therapeutic: In deep vein thrombosis LMWH have been shown to more effective and cause fewer major bleeding complications than heparin[5]. Dalteparin or Enoxaparin in a dose of 100 units per $\mathrm{Kg}$ body weight SC twice a day are recommended. Same dose is recommended in unstable angina. Although considered, as effective as heparin in unstable angina, a recent study has shown reduced incidence of myocardial infarction with LMWH [6].

\section{Adverse effects}

Adverse effects are similar to heparin except that their incidence is reduced. Bleeding, thrombocytopenia and osteoporosis occur less frequently $[2,7]$. Anaphylactoid reaction can occur. Non-surgical bleeding due to overdosage of LMWH is not as easily reversed by protamine as with heparin. In patients of heparin induced thrombocytopenia, LMWH cannot be used because of cross-reactivity. Although they are more costly than heparin, cost advantage of heparin is offset by the cost of hospitalisation and frequent monitoring. With LMWH monitoring of APTT is not recommended except of renal failure and those who have a body weight less than $50 \mathrm{Kg}$ or more than $80 \mathrm{Kg}$. Like heparin LMWH are safe in pregnancy.

\section{Conclusion}

With LMWH, there is convenience in once daily administration of 'fixed dose' for prophylaxis and weight adjusted, un-monitored dose for treatment. Out-patient therapy is safe, efficacious and cost effective. The incidence of bleeding, heparin-induced thrombocytopenia with complicating thromboembolism and osteoporosis is reduced.

\section{REFERENCES}

1. Koopman MMW, Prandoni P, Piovella F et al. Treatment of venous thrombosis with intravenous unfractionated heparin administered in the hospital as compared with subcutaneous low molecular weight heparins administered at home. $\mathrm{N} \mathrm{Engl}$ J Med 1996; 334: 682-7.

2. Weitz Jl, Low molecular weight heparins. N Engl J Med 1997;338: 688-98.

3. Hirsh J, van Atken WG, Gallus AS. Dolleey CT, Cade JF. Yung WL. Heparin kinetics in venous thrombosis and pulmonary embolism. Circulation 1976:53:691-5.

4. Imperiale TF, Speroff T. A meta-analysis of methods to prevent venous thromboembolism following total hip replacements. JAMA 1994;271:1780-5.

5. Lensing AW, Prins MH, Davidson BL. Hirsh J. Treatment of deep vein thrombosis with low molecular weight heparins. A meta-analysis. Arch Intern Med 1995: 155: 601-7.

6. Cohen M, Demeis C, Gurfinkel EP et al. A comparison of low molecular weight heparins with unfractionated heparins in unstable coronary artery disease. N Engl J Med 1997; 338: 447-5.

7. Warkentin TF, Levine $\mathrm{MN}$, Hirsh J et al. Heparin induced thrombocytopenia in patients treated with low molecular weight heparins or unfractionated heparins. N Engl J Med 1995;332: 1330-5. 